\title{
Spirometry in primary care: is it good enough to face demands like World COPD Day?
}

\author{
T. Schermer*, T. Eaton" ${ }^{\#}$ R. Pauwels", C. van Weel*
}

On November 19th this year, World Chronic Obstructive Pulmonary Disease (COPD) Day 2003, people worldwide will be encouraged to review their respiratory health status and consult a doctor in case of certain symptoms [1]. Spirometry would be regarded as an integral component of this consultation. Additionally, asymptomatic smokers $>40$ yrs will be advised to have their lung function checked [1]. Thus, a likely and desirable outcome of World COPD Day could be a considerable and perhaps dramatic increase in demand for spirometry. This is a potentially daunting prospect with important implications in terms of the availability and utilisation of healthcare resources. It is therefore not only timely but essential to reflect on the current status of spirometry in primary care.

Thus far, a New Zealand study which was reported in 1999 presents the only, but extremely welcome, randomised prospective evaluation of the implementation of spirometry in primary care practice formally assessing both the impact of training and quality assurance [2]. The results of this study should be placed in the context of the growing prevalence of COPD which presents an increasing burden on healthcare resources globally [3]. An essential requirement would seem to be the development of high quality spirometry by family physicians on a large scale. The implications are sobering. Family physicians already diagnose 5-10 new cases annually [4], a figure expected to increase in the coming decades. This figure, albeit dramatic, still underestimates the true challenge of COPD. The burden of the disease in the community is much higher and for a substantial number of patients COPD remains undiagnosed and consequently untreated [5]. Cigarette smoking remains the leading cause of COPD and despite heightened public awareness and smoking cessation initiatives, a significant impact on global COPD numbers is not expected in the short or medium term. World COPD Day aims to promote public awareness of COPD. It is to be hoped that early diagnosis of COPD will facilitate the prevention of further damage to the airways and lungs, predominantly by focusing on smoking cessation.

\section{Interface between primary and secondary care}

A crucial initial success of the Global Initiative for Obstructive Lung Disease (GOLD) has been the establishment of a working relationship with primary care, with the involvement of the World Organization of Family Doctors

\footnotetext{
*Dept of General Practice, University Medical Centre Nijmegen,

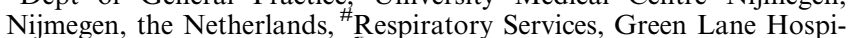
tal, Auckland, New Zealand, "Dept of Respiratory Diseases, University Hospital Ghent, Ghent, Belgium.

Correspondence: T. Schermer, Dept of General Practice 229/HSV, University Medical Centre Nijmegen, PO Box 9101, 6500 HB Nijmegen, the Netherlands. Fax: 31 243541862. E-mail: T.Schermer@, hag.umen.nl
}

(WONCA) [6] and the International Primary Care Respiratory Group (IPCRG) [7]. Early next year the first global primary care guidelines for COPD, based on GOLD, will be published: the International Primary Care Airways Guidelines (IPAG) project [8], developed in cooperation with the World Health Organization (WHO). The introduction of these guidelines will substantially coordinate diagnosis and treatment of COPD in primary care. Although a welcome and valuable initiative, the guidelines do not by themselves address the critically important issue of ensuring that family physicians and other primary care professionals have ready access, not just to spirometry but to quality spirometry.

\section{Spirometry quality assurance}

The impact of World COPD Day is expected to be considerably diminished if spirometry is not widely available and accessible. However, more importantly, poorly performed spirometry may lead to "misdiagnosis" with consequent misdirection of precious healthcare resources and giving rise to unnecessary patient concern. The pivotal role of family physicians dictates that ideally spirometry should be directly available in "every" practice. However, spirometry on this scale does present considerable logistic challenges. Resource and training constraints have for a long time hampered the large-scale introduction of spirometry to primary care. Research and development projects have emphasised rigorous training and performance standards as essential prerequisites of a successful spirometry programme $[2,9]$. Furthermore, longer term "maintenance of standards" is crucial. It is salutary that even under the strict conditions of the Lung Health Study it was observed that the performance of certified technicians with regard to spirometry fell over time [9]. In primary care, spirometry is often, wrongly, regarded as a noninvasive simple screening test. However, it is apparent that careful consideration needs to be given to a number of aspects including selection and maintenance of equipment, optimal performance of the test by both patient and operator, adherence to standard criteria for acceptability and repeatability, appropriate selection of normal predicted values, and careful and informed interpretation of the results [10]. The newer generation electronic spirometers facilitate the adoption of acceptability and reproducibility criteria in primary care, but this should not engender complacency.

While well-established criteria for acceptability and reproducibility have been widely disseminated, it is by no means certain that these are adhered to in clinical practice. The aforementioned New Zealand study of spirometry by family physicians highlights some of these issues and possible solutions [2]. Although a significant training effect was demonstrated, the quality of the spirometry performed by family physicians did not generally satisfy full American Thoracic Society (ATS) criteria for acceptability and reproducibility 
[11]. However, the ATS guidelines allow for the use of data from unacceptable or nonreproducible manoeuvres at the discretion of the interpreter. Since most of the failures seen in primary care spirometry appear to be end-of-test related [2, 12] the forced expiratory volume in one second (FEV1) may still be valid for screening purposes. Formal laboratory-based diagnostic lung function testing could subsequently confirm abnormal screening findings and identify the inevitable falsepositive cases. There is little doubt that poorly performed spirometry leads to increased inappropriate referral to chest physicians, particularly when end-of-test criteria are not met [2]. This serves only to further reinforce the importance of adequate training and quality assurance for successful spirometry in primary care.

\section{Organising spirometry in primary care}

The British Thoracic Society guidelines for COPD [13] acknowledge that healthcare planners may need to consider options for the provision of primary care spirometry other than having the appropriate equipment on-site. Alternatives could include utilising primary care diagnostic services or hospital-based laboratories, although there may be certain disadvantages, including barriers to access (table 1) [10]. Furthermore, relevant spirometric indices measured by trained family physicians or their staff may be marginally higher compared with pulmonary function laboratories [14]. In view of the limited agreement between laboratory and primary care practice FEV1 and forced vital capacity values, these measurements should not be used interchangeably [14].

The bottom line is that with sufficient training of family physicians and their staff, the current practice of performing spirometry in primary care seems justifiable. However the experience serves as a valuable insight into the clinical reality of performing spirometry on a larger scale in primary care practice. It is envisaged that the optimal method of ensuring quality spirometry will entail a close partnership between primary healthcare providers and specialised respiratory care. This integrated approach with specialist respiratory services is to be recommended, but it is unrealistic to expect this to become the state of the art universally overnight. The true challenge is to build the required infrastructure, in terms of equipment resource and adequate training and expertise. Although the magnitude of this challenge should not be underestimated, the published evidence $[2,14]$ points the way to widespread implementation of spirometry in primary care. The community of respiratory health professionals is at the start of a journey; the goals being to address the global burden of COPD as outlined by GOLD. Spirometry by family physicians, with due consideration to quality assurance, is quintessential to this process.

\section{Conclusions}

Spirometry, in conjunction with a commitment to smoking cessation initiatives, has the exciting potential to impact significantly on global health. Given the partnership with primary care that the Global Initiative for Obstructive Lung Disease (GOLD) has established, and capitalising on the experience of family physicians the priority now is the development of accessible primary care spirometry around the world, working in close cooperation with specialised respiratory facilities to facilitate quality assurance. World Chronic Obstructive Pulmonary Disease Day is a valuable and important initiative and will serve to mobilise global action on chronic obstructive pulmonary disease. However its

Table 1.-Advantages and disadvantages of different alternatives for organising spirometry in primary care

Where spirometry performed Advantages Disadvantages

General practice surgery

Nurse-run asthma/COPD clinic

PCG-commissioned spirometry service

Hospital-based pulmonary function laboratory
Least barriers to access

No extra healthcare costs

Least travelling distance for patients

Minimises number of patient visits ("one-stop shop")

Results of spirometry integrated into first consultation

Enables FPs to acquire expertise

Good reliability of measurements

Few access limitations

No extra workload for family practices

No high demands on spirometry expertise in family practices

Good reliability of measurements

Few access limitations

No extra workload for family practices

No high demands on spirometry expertise in family practice

Centralisation of interpretation of spirometry

Optimum reliability of measurements

No extra workload for family practices

No high demands on expertise in family practice

Facilitates consultation of specialist respiratory services \#
Reliability of measurements less certain Extra workload for family practices Family practice has to build up expertise Changes in practice organisation (often) necessary

Extra healthcare costs

(Considerable) travelling distance for patients

Timely feedback of spirometry

results to family practice crucial

Extra healthcare costs

(Considerable) travelling distance for patients

Timely feedback of spirometry

results to family practice crucial

Possible access limitations ${ }^{\#}$

Limited capacity next to regular tasks

Extra healthcare costs

(Considerable) travelling distance for patients

Timely feedback of test results to family practice crucial 
true potential will only be achieved by ensuring that quality spirometry is widely available in primary care. This is a vital prerequisite to the success of both World Chronic Obstructive Pulmonary Disease Day and the larger Global Initiative for Obstructive Lung Disease (GOLD) strategic plan. Never before have all parties involved been better positioned to contribute towards this collective goal.

\section{References}

1. Global Initiative for Chronic Obstructive Lung Disease ${ }^{\mathrm{TM}}$. www.goldcopd.com. Date accessed: June 122003.

2. Eaton T, Withy S, Garrett JE, Mercer J, Whitlock RML, Rea HH. Spirometry in primary care practice. The importance of quality assurance and the impact of spirometry workshops. Chest 1999; 116: 416-423.

3. Rutten-van Molken MP, Postma MJ, Joore MA, van Genugten ML, Leidl R, Jager JC. Current and future medical costs of asthma and chronic obstructive pulmonary disease in The Netherlands. Respir Med 1999; 93: 779-787.

4. Tirimanna PRS, van Herwaarden CLA, van Schayck CP, et al. Prevalence of asthmas and COPD in general practice in 1992: has it changed since 1977? Br J Gen Pract 1996; 46: 277-281.

5. van Weel C. Underdiagnosis of asthma and COPD: is the general practitioner to blame? Monaldi Arch Chest Dis 2002; 57: $65-68$

6. Global Family Doctor - WONCA Online. Website of the
World Organization of Family Doctors. www.globalfamilydoctor.com. Date accessed: June 92003.

7. IPCRG - International Primary Care Respiratory Group www.ipcrg.org Date updated: July 30 2003; Date accessed: May 72003.

8. Korsten AMMH, van Schayck CP. New International Primary Care Airways Guidelines (IPAG). Revue Française d' Allergologie et d' Immunologie Clinique 2003; 43: 246-248.

9. Enright PL, Johnson LR, Connett JE, Voelker H, Buist AS. Spirometry in the Lung Health Study. 1. Methods and quality control. Am Rev Respir Dis 1991; 143: 1215-1223.

10. Schermer TRJ, Folgering HTM, Bottema BJAM, Jacobs JE, van Schayck CP, van Weel C. The value of spirometry for primary care: Asthma and COPD. Prim Care Respir J 2000; 9: 51-55.

11. American Thoracic Society. Standardization of spirometry: 1994 update. Am J Respir Crit Care Med 1995; 152: 1107-1136.

12. den Otter JJ, Knitel M, Akkermans RP, van Schayck CP, Folgering HT, van Weel C. Spirometry in general practice: the performance of practice assistants scored by lung function technicians. Br J Gen Pract 1997; 47: 41-42.

13. The COPD Guidelines Group of the Standards of Care Committee of the BTS. BTS Guidelines for the management of chronic obstructive pulmonary disease. Thorax 1997; 52: Suppl. 5, S1-28.

14. Schermer T, Jacobs A, Chavannes N, Hartman J, Folgering $\mathrm{H}$, Bottema B, van Weel C. Validity of spirometry in a general practice population of patients with chronic obstructive pulmonary disease (COPD). Thorax 2003 (in press). 